\title{
ALTERAÇÕES FISIOLÓGICAS EM CUPUAÇUZEIROS SADIOS E INFECTADOS POR VASSOURA DE BRUXA
}

\author{
SILVA JUNIOR, João Fernandes da ${ }^{1}$ \\ SOBRINHO, Rômulo José Alencar ${ }^{2}$ \\ FRANÇA, Sueny Kelly Santos de ${ }^{2}$ \\ REIS, Iolanda Maria Soares ${ }^{1}$ \\ PEREIRA, Gener Tadeu ${ }^{3}$
}

\begin{abstract}
RESUMO: O cupuaçu [Theobroma grandiflorum (Willd. ex Spreng.)Schum] é uma das frutas mais importantes e populares da Amazônia. Porém o cultivo ainda é reduzido devido poucas informações agronômicas e falta de material genético selecionado, além dos danos causados pela "vassoura de bruxa" são responsáveis pela baixa produção. O presente estudo teve como objetivo avaliar alguns parâmetros fisiológicos em cupuaçuzeiros sadios e infectados com vassoura de bruxa. Este estudo foi conduzido em cupuaçuzeiros de 10 anos de idade consorciada com seringueira (Hevea brasiliensis) com 25 anos de idade no Campus da Universidade Federal Rural da Amazônia, em Belém-PA. Foram medidos com o auxilio de um porômetro de difusão de estado estacionário, marca LI-COR, Modelo 1600, para mensurar as seguintes variáveis: Condutância Estomática (CE); Radiação Fotossinteticamente Ativa (R.F.A); Temperatura da folha (T) e Transpiração (Tr);Umidade Relativa da Folha. Para a determinação dos parâmetros foram utilizadas 10 plantas sadias e 10 plantas inoculadas com o fungo Crinipellis perniciosa. Medindo com o porômetro na superfície abaxial de uma folha no terço mediano da copa de cada planta medidas a partir do dia 28/01/2006, em 4 dias consecutivos nos períodos de $8 \mathrm{~h}, 10 \mathrm{~h}, 12 \mathrm{~h}, 14 \mathrm{~h}$ e $16 \mathrm{~h}$. Com base nos dados registrados, aplicou-se o teste de tukey a $5 \%$. E as plantas atacadas com a vassoura de bruxa apresentaram menor transpiração e condutância estomática comparando com as plantas sadias. Sendo que houve diferença significativa apenas nos períodos de $10 \mathrm{~h}$ às $14 \mathrm{~h}$. Isto implicou diretamente na diminuição da fotossíntese e consequentemente na produtividade da cultura.
\end{abstract}

Palavras-chave: Condutância estomática. Theobroma grandiflorum. Crinipellis perniciosa.

\section{CHANGES IN PHYSIOLOGICAL CUPUAÇUZEIROS HEALTHY AND INFECTED BY THE WITCH BROOM}

SUMMARY: The Cupuassu [Theobroma grandiflorum (Willd. ex Spreng.) Schum] is one of the most
important fruit and popular in the Amazon. But cultivation is still limited because few agronomic information
and lack of selected genetic material, in addition to the damage caused by "witches broom" are responsible for
the low production. This study aimed to evaluate physiological parameters of healthy Cupuassu and infected
with witches' broom. This study was conducted in Cupuassu 10 years of age intercropped with rubber (Hevea
brasiliensis) with 25 years of age on the Campus of Federal University Rural of Amazonia, Belém-PA. Were
measured with the aid of a diffusion porometer state stationary, brand LI-COR, Model 1600, to measure the
following variables: stomatal conductance (EC); Photosynthetically Active Radiation (PAR), leaf temperature
(T) and transpiration (Tr) of Relative Humidity Sheet. To determine the parameters used were 10 healthy plants
and plants inoculated with 10 fungus $C$. perniciosa. Measuring with the porometer on abaxial surface of a leaf in
the middle third of crown of each plant measured from the day 01.28 .2006 on 4 consecutive days during periods
of $8 \mathrm{~h}, 10 \mathrm{~h}, 12 \mathrm{~h}, 14 \mathrm{~h}$ and $16 \mathrm{~h}$. Based on the recorded data, we applied the Tukey test at $5 \%$. And the plants
infested with the witches' broom disease had lower stomatal conductance and transpiration compared to the

${ }^{1}$ Eng $^{\text {o Agro }}$. Mestrando do Curso de Pós-Graduação em Agronomia (Ciência do Solo). Faculdade de Ciências
Agrárias e Veterinárias (FCAV), UNESP - Universidade Estadual Paulista, Campus de Jaboticabal. Bolsista
CAPES. E- mail: joaojrsilva@ @ahoo.com.br
${ }^{2}$ Eng $^{\circ}$ Agr ${ }^{\circ}$. Mestrando do Curso de Pós-Graduação em Solos e Nutrição de Plantas. UFRA. Bolsista do CNPq.
${ }^{3}$ Estatístico. Professor Adjunto. FCAV, UNESP, Campus de Jaboticabal, Departamento de Ciências Exatas. 
healthy plants. And there was a significant difference only in periods of $10 \mathrm{~h}$ and $14 \mathrm{~h}$. This involved directly in decrease in photosynthesis and consequently the productivity.

Keywords: Stomatal Conductance. Theobroma grandiflorum. Crinipellis perniciosa.

\section{INTRODUÇÃO}

O cupuaçu [Theobroma grandiflorum (Willd. ex Spreng.)Schum] pertence a família Sterculiaceae é uma das frutas mais importantes e populares da Amazônia. A espécie é uma árvore (Figura 1) que atinge $7 \mathrm{~m}$ de diâmetro de copa, $4 \mathrm{~m}$ a 10m de altura média, com frutos do tipo baga, com formatos variáveis, extremidades obtusas ou arredondadas (Figura 3), diâmetro de $9 \mathrm{~cm}$ a $15 \mathrm{~cm}$, comprimento de $10 \mathrm{~cm}$ a $40 \mathrm{~cm}$, peso variando de $300 \mathrm{~g}$. Seu fruto é o maior entre os dos gêneros, sendo considerado um dos melhores da flora regional, cuja demanda é cada vez mais crescente devido seu sabor agradável e sua rentabilidade como cultura perene (CALZAVARA, 1987). É encontrada desenvolvendo-se espontaneamente nas matas do nordeste da Amazônia Oriental, nordeste do Maranhão, é atualmente disseminado por toda bacia amazônica brasileira.

Atualmente essa fruta começa a despertar interesse dos grandes produtores, pois o seu comercio está sendo expandido para outros estados do Brasil e até do exterior (VENTRIERI, 1993). A partir de sua polpa podem ser elaborados sorvetes, sucos, compotas, geléias, licores, iogurtes e diversos outros doces (CALZAVARA.; MULLER; KAWAGE, 1984; VENTRIERI et al., 1985). As amêndoas fermentadas são utilizadas na fabricação de chocolate conhecido como "cupulate", produto semelhante ao chocolate de cacau (NAZARÉ,; BARBOSA; VIEGAS, 1990). Porém, o cultivo ainda é reduzido devido poucas informações agronômicas e falta de material genético selecionado, além dos danos causados pela "vassoura de bruxa", os quais são responsáveis pela perda de 50 a $60 \%$ em alguns casos até 100\%. De acordo com (SOUZA, 2007) a redução da produtividade observada dos cultivos na região amazônica tem como uma das causas a vassoura-de-bruxa

A vassoura-de-bruxa, Crinipellis (Moniliophthora)perniciosa (Stahel), é a doença que mais ameaça os plantios de cupuaçuzeiros, na Região Amazônica. Encontra-se disseminada, de forma endêmica, em toda a região tropical da América do Sul (PURDY; SCHMIDT, 1996). O ataque ocorre em regiões meristemáticas, como gemas axilares e apicais, promovendo hipertrofia, com brotações vegetativas deformadas e entrenós curtos as quais, após um mês, secam, interferindo significativamente na área fotossintética da planta (ALVES, et al., 2009). 
Segundo Muller et al. (1995), quando submetidos a déficit hídrico, o cupuaçuzeiro apresenta paralisação no crescimento; perdas de folhas; secamento do broto terminal; maior susceptibilidade ao ataque de pragas e doenças; e a morte da planta, conforme a intensidade do déficit hídrico.

Os fungos em contato com as plantas produzem toxinas capazes de inibir a atividade de certas enzimas, que direta ou indiretamente, estão envolvidas no processo fotossintético e consequentemente, podem diminuir a transpiração e a condutância estomática (NUNES,2000).

Em função da carencia de trabalhos de pesquisa com a cultura do cupuaçu, o objetivo deste foi estudar as alterações fisiológicas de cupuaçuzeiros sadios e infectados com fungos de Crinipellis perniciosa (Stahel) em um sistema agroflorestal.

\section{MATERIAL E MÉTODOS}

O experimento foi conduzido em condições de campo na área experimental da Universidade Federal Rural da Amazônia no Campus de Belém, nas coordenadas Latitude $01^{\circ}$ 28' 03”S e Longitude 48 29' 18'E. O clima da região é quente úmido, do tipo Ami na classificação de Koppen. Segundo Bastos (1982), caracteriza-se como clima tropical chuvoso sem variação térmica estacional e apresentando total pluviométrico anual elevado, com mês menos chuvoso inferior a $60 \mathrm{~mm}$ e com moderado período de estiagem.

As formas de relevo preponderante na área experimental revelam topografia plana, com declives entre 0 a 3\%, ligeiramente onduladas. Os solos predominantes na área são do grupo dos Latossolos Amarelos textura média, fortemente lixiviados, com baixa fertilidade natural e o teor de argila aumenta com a profundidade.

Utilizando-se 20 plantas de cupuaçuzeiros de um plantio com 10 anos de idade, consorciados com seringueiras (Hevea brasiliensis M. Arg - clone Fx 3899) com 25 anos de idade, utilizando-se 10 plantas enxertadas sadias e 10 plantas inoculadas com o fungo Crinipellis perniciosa conforme fig. 1. Medindo com o auxílio de um porômetro de difusão de estado estacionário, marca LI-COR, Modelo LI 1600 - C, aclopado a um sensor quântico LICOR, Modelo QUANTUM Q22341, para medição da na superfície abaxial de uma folha totalmente expandidas no terço mediano da copa de cada planta, para mensurar as seguintes variáveis: Condutância Estomática ( CE ); Radiação Fotossinteticamente Ativa ( R.F.A ); Temperatura da folha (T) e Transpiração (Tr);Umidade Relativa da Folha. Sabendo que a primeira foi encontrada a partir da Resistência estomática conforme a equação (1) 


$$
C=\frac{1}{R}
$$

Em que,

$C$ - condutância estomática, mmol. $\mathrm{m}^{-2} \cdot \mathrm{s}^{-1}$

e

$R$ - resistência estomática, $\mathrm{mmol} \cdot \mathrm{m}^{-2} \cdot \mathrm{s}^{-1}$

Medidas a partir do dia 28/01/2006, em 4 dias consecutivos nos períodos de $8 \mathrm{~h}, 10 \mathrm{~h}$, $12 \mathrm{~h}, 14 \mathrm{~h}$ e $16 \mathrm{~h}$.

Mediu-se ainda, a temperatura ambiente e umidade relativa do ar através de um termômetro digital. Com base nos dados registrados, foram submetidos à análise de variância e aplicou-se o teste de tukey a 5\% de acordo com Banzatto e Kronka (2006).
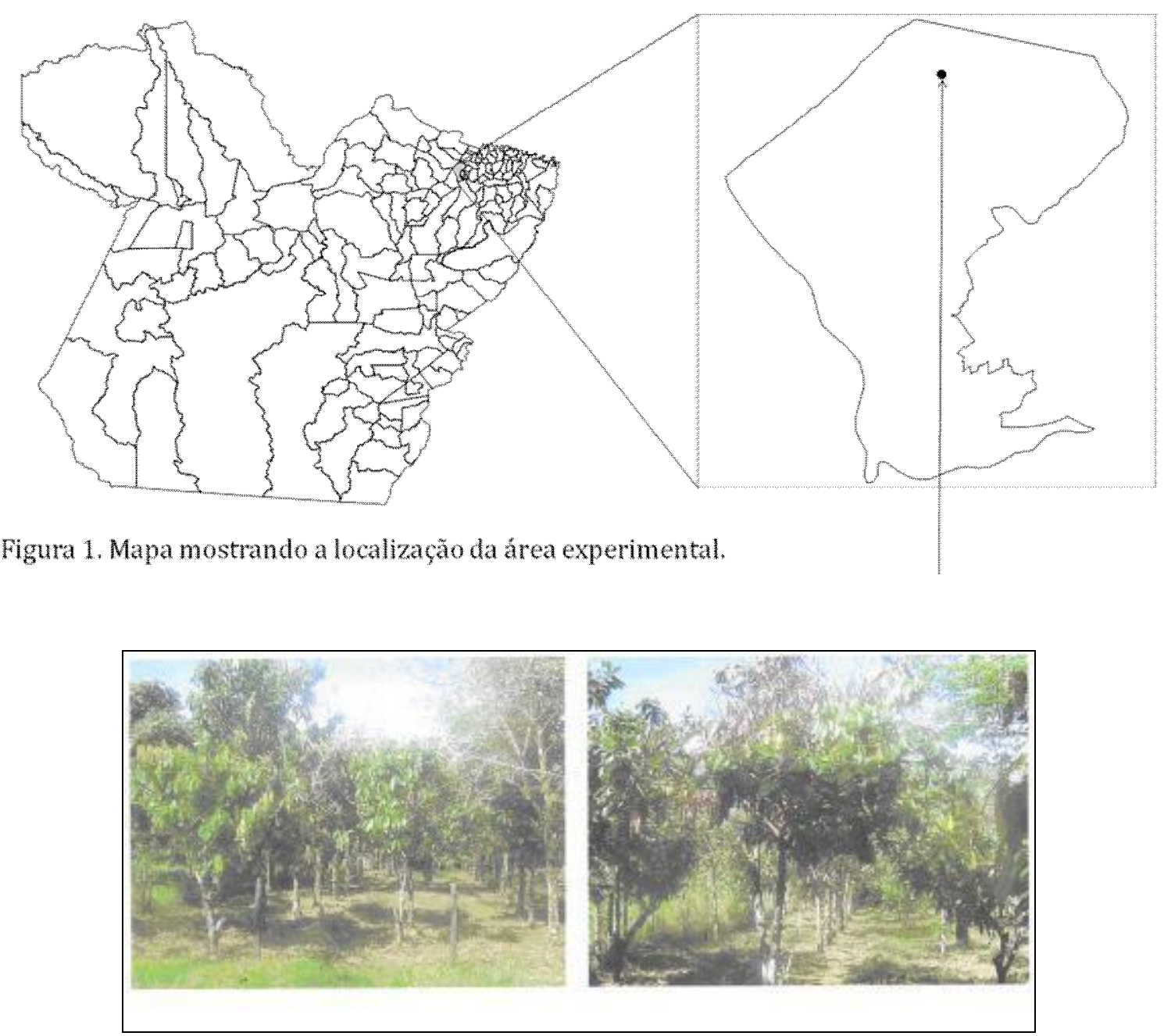

Figura 2. Cupuaçuzeiros sadios

Cupuaçuzeiros infectados 


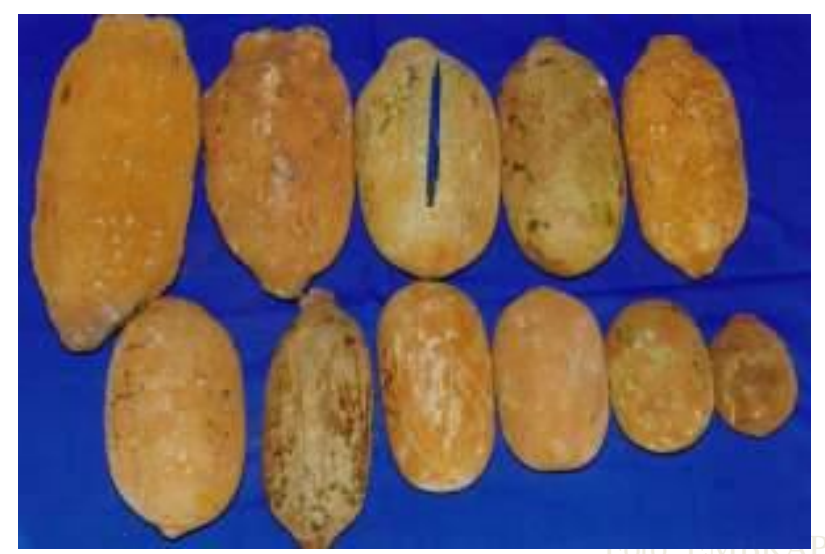

Figura 3. Frutos de cupuaçu com formas variadas

\section{RESULTADOS E DISCUSSÃO}

Verifica-se na Figura 4 que as plantas sadias apresentaram maior condutância estomática em relação às infectadas entre 8 h e 14 h, período de maior intensidade luminosa.

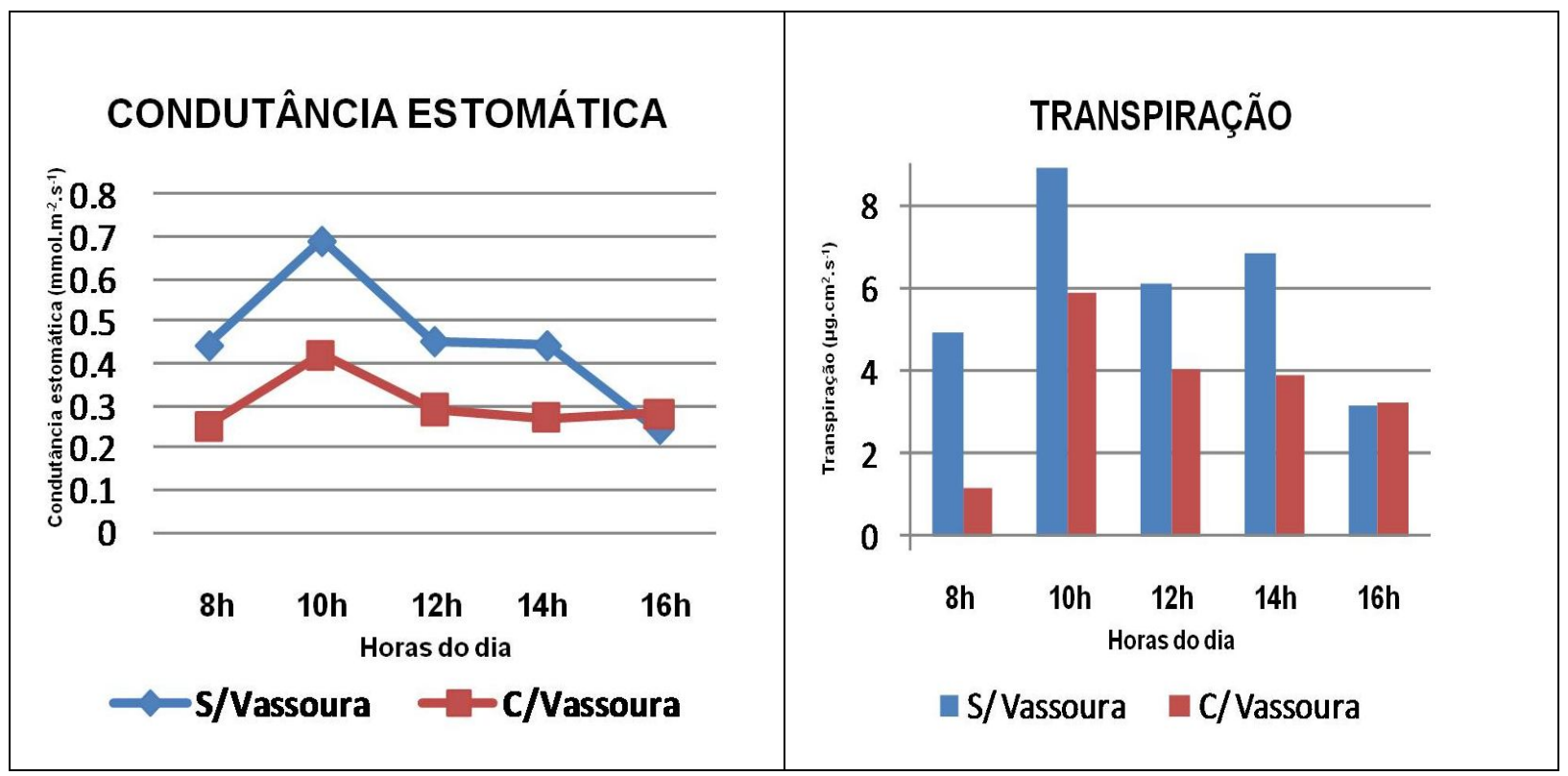

Figura 4. Condutância estomática e Transpiração em relação aos períodos de medidos.

Relacionando-se a condutância estomática com a radiação fotossinteticamente ativa (Figura 5). Observa se que apesar da R.F.A ser alta por volta das 12 h, a condutância estomática em ambas as plantas decresceram, fenômeno esse que segundo Taiz e Zeiger (2004), plantas submetidas a altas temperaturas começam a estabilizar-se alcançando a saturação, e em resposta a isso a planta fornece meios efetivos para controlar a perda de água 
e absorção de $\mathrm{CO} 2$ aumentando a resistência dos estômatos. Como a resistência estomática é inversamente proporcional a condutância estomática a última tende a diminuir a medida que aumenta a resistência dos estômatos. Corroborando com o trabalho com mogno de Dias ; Marenco (2007).

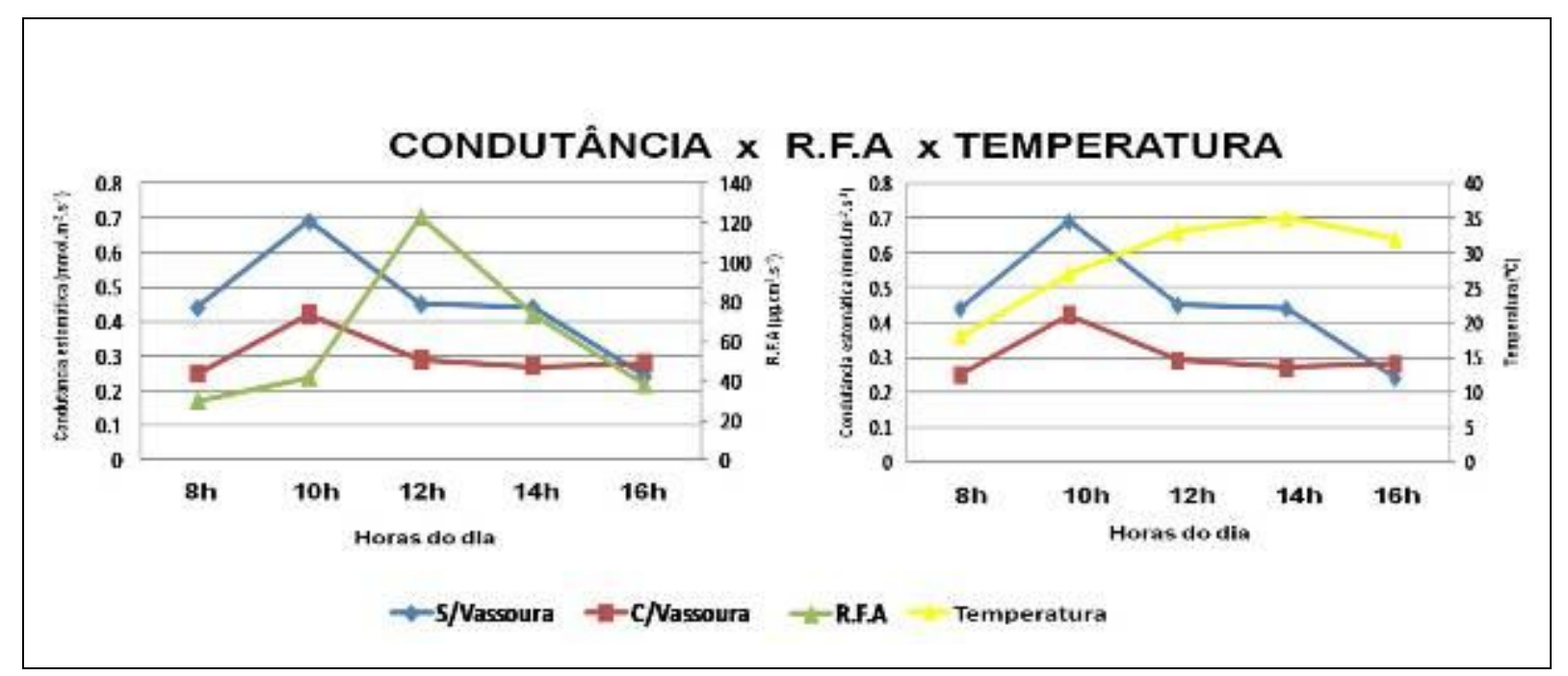

Figura 5. Condutância estomática, R.F.A e Temperatura em relação aos períodos medidos.

A diminuição da umidade relativa do ar e aumento da temperatura Tabela 1. Nesse período mais quente pode ter influenciado no fechamento dos estômatos. Pois, de acordo com Taiz e Zeiger, (2004), quando existe uma diferença de potencial hídrico no sistema SoloPlanta-Atmosfera há perda de água pela planta para atmosfera intercambiando água por produtos da fotossíntese, sendo essenciais para o crescimento e reprodução. Porém, o que deve ter acontecido é que o solo é menos abundante em água, a planta mantém seus estômatos fechados para evitar desidratação, aumentando a resistência estomática e aumentando a transpiração em todas as plantas analisadas.

Tabela 1. Temperatura ambiente e umidade relativa do ar nos diferentes períodos medidos

\begin{tabular}{clcccc}
\hline Horário & $8 \mathrm{~h}$ & $10 \mathrm{~h}$ & $12 \mathrm{~h}$ & $14 \mathrm{~h}$ & $16 \mathrm{~h}$ \\
\hline Temperatura & 28 & 30,5 & 34,2 & 34,5 & 30,2 \\
U.R. Ar $(\%)$ & $>90$ & 74 & 64 & 64 & 65 \\
\hline
\end{tabular}

A redução da transpiração nas plantas com vassoura de bruxa é notavelmente observada na fig.6. Isso devido às quedas das folhas com o efeito da doença ao longo dos anos. Falcão; Morais; Clemente. (1998) também encontraram essas mudanças foliares, que interferiram na transpiração, floração e frutificação de cupuaçuzeiros atacados com o fungo Crinipellis perniciosa. 


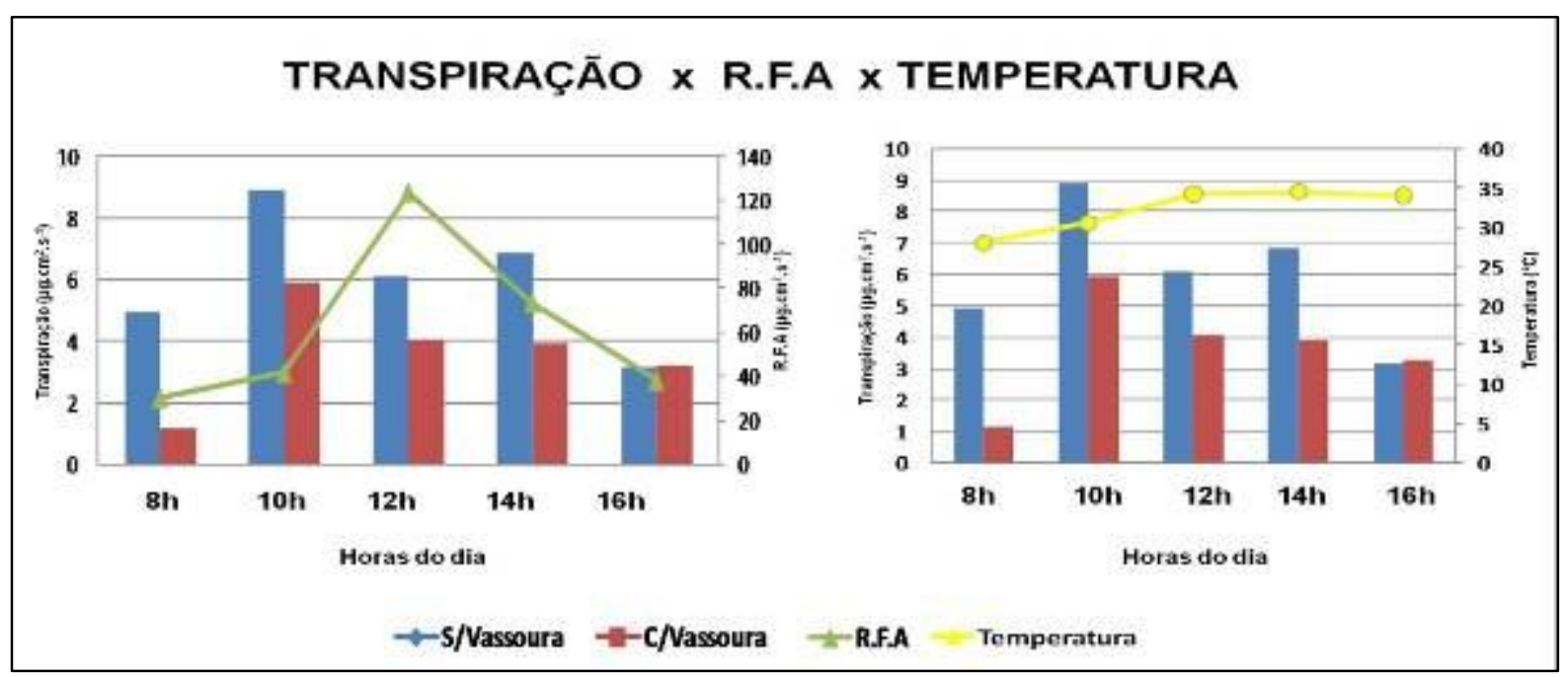

Figura 6. Alterações da Transpiração com a R.F.A e a Temperatura em relação aos períodos medidos.

As alterações na transpiração das plantas atacadas também pode ser explicada devido as condições climáticas da região, a qual favorecem a doença, além de que, não foram realizadas podas fitossanitárias. Motivo segundo Venturieri et al. (1993). Pode influenciar na severidade da doença em relação às alterações da fisiologia da planta, reduzindo significativamente a produção econômica da cultura.

\section{CONCLUSÃO}

As plantas infectadas apresentaram menor transpiração e condutância estomática em relação às sadias.

O fungo Crinipellis perniciosa é limitante para o desenvolvimento da cultura do cupuaçu, sobretudo do ponto de vista dos parâmetros fisiológicos estudados neste trabalho.

\section{REFERÊNCIAS}

ALVES, R. M. Evolução da vassoura-de-bruxa e avaliação da resistência em progênies de cupuaçuzeiro. Rev. Bras. Frutic., Jaboticabal, v. 31, n. 4, p. 1022-1032, dez. 2009.

BANZATTO, D.A; KRONKA, S.N. Experimentação agrícola. 4.ed. Jaboticabal: FUNEP, 2006. 236 p.

BASTOS, T. X. O clima da Amazônia brasileira segundo Koppen. Belém: EMBRAPACPATU, 1982. 4 p.

CALZAVARA, B. B. G.; MULLER, C. H.; KAWAGE, O. N. C. Fruticultura tropical: o cupuaçuzeiro: cultivo, beneficiamento e utilização do fruto. Belém : EmbrapaCPATU,1984. 101 p. (Embrapa-CPATU. Documentos, 32). 
CALZAVARA, B. B. G. Recomendações básicas. Belém: EMBRAPA CPATU,1987.

DIAS, D.P.; MARENCO, R.A. Fotossíntese e fotoinibição em mogno e acariquara em função da luminosidade e temperatura foliar. Pesq. Agrop. Bras, v.42, n.3, p. 305-311, 2007.

FALCÃO, M.A; MORAIS,R. R.; CLEMENTE, C. R. Influência da vassoura de bruxa na fenologia do cupuaçuzeiro: Acta Amazônica, v.29, n.1, p.13-19, 1999.

MULLER, C. H. et al. A cultura do cupuaçu. Brasília, DF: Embrapa-SPI; Belém: EmbrapaCPATU, 1995, 61 p. (Embrapa -SPI. Coleção Plantar, 24; Série Vermelha. Fruteiras).

NAZARÉ, R.F.R de; BARBOSA, W.C; VIEGAS.R.M.F. Processamento das sementes de cupuaçu para obtenção de cupulate. Belém: EMBRAPA/CPATU, 1990. 38p. (Boletim de Pesquisa n.108).

NUNES, M. A. L. Comportamento ecofisiológico e fitopatológico de bananeiras (Musa spp') em solo artificialmente enfestado com Fusarium oxysporum f.sp. cubense (E.F. Smith) sn. E Hansen, nas condições da Amazônia Oriental. Belém-PA, 2000.

PURDY, L. H.; SCHMIDT, R.A. Status of cacao witches' broom: biology, epidemiology and management. Annual Review Phytopathology. Wardowsky, v.34, p.573-94, 1996.

SOUZA, A. G. C. Boas práticas agrícolas da cultura do cupuaçuzeiro. Manaus: Embrapa Amazônia Oriental, 2007. 56 p.

TAIZ, L.; ZEIGER, E. Fisiologia vegetal. Porto Alegre: Artmed, 2004. p.83-92.

VENTURIERI, G. A. Cupuaçu: a espécie, sua cultura, usos e processamentos. 1993. 108 p.

VENTURIERI, G. A.; ALVES, M. L. B.; NOGUEIRA, M. Q. O Cultivo do cupuaçuzeiro. Informativo SBF, Itajaí, v. 4, n. 1, 1985. 